\title{
DISSECÇÃO ESPONTÂNEA DAS ARTÉRIAS CARÓTIDAS E VERTEBRAIS EM UMA POPULAÇÃO MULTIÉTNICA
}

\author{
Alexandre Pieri', Mariana Spitz', Raul Alberto Valiente', \\ Wagner Mauad Avelar', Gisele Sampaio Silva', Ayrton Roberto Massaro'
}

\begin{abstract}
RESUMO - A dissecção espontânea das artérias carótidas e vertebrais (DEACV) é considerada uma causa rara de acidente vascular cerebral, particularmente em países com população multiétnica. O objetivo desse estudo foi avaliar características clínicas e de neuroimagem dos pacientes com DEACV em uma população multiétnica. Foram estudados 66 pacientes com diagnóstico de DEACV em dois hospitais terciários de São Paulo. Aplicou-se um questionário inicial e os pacientes foram seguidos prospectivamente. Dos pacientes estudados, $82 \%$ eram brancos, $53 \%$ eram homens e a média de idade foi 41,7 anos. Os fatores de risco cardiovasculares mais freqüentes foram hipertensão arterial e tabagismo. Outros aspectos avaliados foram história prévia de enxaqueca, tratamento inicial e prognóstico. Concluiu-se que apesar da população estudada ser multiétnica, houve um marcante predomínio de brancos. A análise das características clínicas e de neuroimagem dos pacientes com DEACV possibilita um melhor conhecimento da doença, levando a um diagnóstico precoce e tratamento mais adequado.
\end{abstract}

PALAVRAS-CHAVE: dissecação da artéria vertebral, dissecação da artéria carótida interna, acidente cerebrovascular, grupos étnicos.

\begin{abstract}
Spontaneous carotid and vertebral arteries dissection in a multiethnic population
ABSTRACT - Spontaneous dissection of the carotid and vertebral arteries (SDCVA) is considered a rare cause of stroke, particularly in countries with multiethnic population. The objective was to evaluate the clinical and neuroimaging features of patients with SDCVA from a multiethnic population. Sixty-six patients diagnosed with SDCVA were studied at two tertiary hospitals at São Paulo. An initial questionnaire was completed and patients were followed prospectively. Among the patients studied, $82 \%$ were caucasian, $53 \%$ were male and the average age was 41.7 years old. The most frequent cardiovascular risk factors found were systemic hypertension and tobacco use. Other aspects evaluated were history of previous migraine, initial treatment and prognosis. In conclusion, although the population studied was multhiethnic, there was a marked predominance of caucasians. The analysis of clinical and neuroimaging data from patients with SDCVA allows a better understanding of the disease, leading to an earlier diagnosis and more appropriate treatment.
\end{abstract}

KEY WORDS: vertebral artery dissection, carotid artery internal dissection, cerebrovascular disorder, ethnic groups.

A dissecção espontânea das artérias carótidas e vertebrais (DEACV) caracteriza-se pela sua ruptura não traumática, entre as camadas arteriais, levando à redução do diâmetro da luz da artéria ou à formação de dilatação aneurismática'. Atualmente é considerada uma das causas mais comuns de acidente vascular cerebral (AVC) em adultos jovens ${ }^{2}$ e apresenta grande variabilidade na forma de apresentação clínica. Com o desenvolvimento das técnicas angiográficas, houve aumento no número de casos de DEACV diagnosticados, permitindo melhor compreensão desta condição $0^{3,4}$. Apesar disso, a DEACV continua sendo uma doença subdiagnosticada ${ }^{5}$. Novos estudos são necessários para o esclarecimento de dúvidas que ainda persistem quanto à etiologia, diagnóstico, tratamento e prognóstico dos pacientes com DEACV, especialmente em populações multiétnicas.

O objetivo deste estudo foi descrever, a partir de uma série de casos, as características clínicas e os achados de neuroimagem mais freqüentemente observados nos pacientes com DEACV em uma população hospitalar brasileira.

\section{MÉTODO}

Estudo de série de casos, bicêntrico, prospectivo, com um total de 66 pacientes com diagnóstico confirmado de

'Setor de Neurovascular, Departamento de Neurologia, Universidade Federal de São Paulo, São Paulo, Brasil; ${ }^{2}$ Hospital Santa Marcelina, São Paulo, Brasil

Recebido 19 Abril 2007, recebido na forma final 28 Junho 2007. Aceito 10 Agosto 2007.

Dr. Alexandre Pieri - Rua Napoleão de Barros 874 / 14 - 04024 -002 São Paulo SP - Brasil. 
DEACV, atendidos nos serviços de Neurologia Vascular do Hospital São Paulo (Escola Paulista de Medicina, Universidade Federal de São Paulo - Unifesp) e do Hospital Santa Marcelina, dois hospitais terciários de ensino da região metropolitana do município de São Paulo, Brasil. O estudo foi realizado no período de maio de 2001 a junho de 2006 .

Todos os pacientes assinaram um termo de consentimento livre e esclarecido e o projeto foi aprovado pelo Comitê de Ética em Pesquisa da Universidade Federal de São Paulo.

Após a alta hospitalar, todos os pacientes foram encaminhados ao ambulatório de Neurologia Vascular do Hospital São Paulo, onde foram reavaliados trimestralmente.

Foram incluídos pacientes com sinais e sintomas compatíveis com o diagnóstico clínico presuntivo de DEACV e confirmação neurorradiológica de DEACV pela angio-ressonância (angioRM) de encéfalo e/ou angiografia cerebral com cateterismo, ou através da demonstração do "sinal da dupla luz" na ressonância magnética (RM) de encéfalo com cortes finos axiais em T1 com supressão de gordura.

Foram excluídos pacientes com confirmação ou suspeita de dissecção associada a doença aterosclerótica e/ou trauma, podendo este ser decorrente tanto de eventos específicos, como acidente de trânsito, lesão por arma branca ou de fogo, como de iatrogenia relacionada a procedimentos diagnósticos ou cirurgia cervical.

As informações clínicas dos pacientes foram coletadas por meio de uma ficha pré-estabelecida contendo dados demográficos, fatores de risco cardiovasculares, fatores associados, quadro clínico de apresentação, diagnóstico por neuroimagem, tratamento e prognóstico. Os dados complementares, referentes ao seguimento dos pacientes, foram obtidos nas consultas médicas subseqüentes.

Dados demográficos - O grupo étnico dos pacientes foi determinado com base nas características fenotípicas e mediante auto-identificação. Os pacientes foram classificados como brancos, afro-brasileiros (negros e mulatos) e asiáticos. Foram coletados ainda dados referentes à idade e sexo dos pacientes.

Fatores de risco cardiovasculares - Quanto aos fatores de risco cardiovasculares, avaliou-se história prévia de hipertensão arterial sistêmica (HAS), diabetes, dislipidemia, tabagismo e consumo de bebidas alcoólicas. A presença de HAS, diabetes e dislipidemia foi caracterizada segundo consenso previamente publicado ${ }^{6}$. Foram considerados etilistas todos os pacientes que referiram hábito de ingerir bebidas alcoólicas contendo o equivalente a $75 \mathrm{~mL}$ de etanol por dia7.

Fatores predisponentes - Fatores predisponentes como história prévia de enxaqueca, infecção recente e evento menor precipitante não traumático também foram investigados. Enxaqueca foi definida de acordo com os critérios diagnósticos da Classificação Internacional das Cefaléias ${ }^{8}$. Considerou-se evento menor precipitante como movimento de rotação lateral, flexão ou extensão cervical decorrente de atividade cotidiana, não associado a trauma 9 . Como infecção recente, foram consideradas as infecções de vias aéreas superiores e inferiores que ocorreram até 1 mês antes da DEACV ${ }^{10}$.
Quadro clínico - Foram obtidas informações sobre sinais e sintomas neurológicos no início da DEACV. O tempo de chegada dos pacientes ao hospital foi determinado e a gravidade do quadro à admissão foi avaliada através da aplicação da escala de AVC do $\mathrm{NIH}^{11}$. A presença de sinais e sintomas de alerta como cefaléia, síndrome de Horner e paralisia de nervos cranianos foi verificada. O quadro clínico dos pacientes com dissecção de artéria carótida (DAC) e com dissecção de artéria vertebral (DAV) foi avaliado separadamente, em decorrência das particularidades dos diferentes territórios arteriais. Quanto à forma de apresentação clínica à admissão, houve uma subdivisão em pacientes com presença de evento isquêmico, hemorrágico ou sem evento neurovascular associado.

Diagnóstico por neuroimagem - Os pacientes com DEACV foram submetidos a exames de neuroimagem no momento do diagnóstico: ultra-sonografia (USG) com Doppler de artérias cervicais, tomografia computadorizada (TC) de crânio, RM e angiografia por ressonância (angioRM) ou com cateterismo. Os exames foram realizados nos serviços de Radiologia do Hospital Santa Marcelina e do Hospital São Paulo.

Tratamento - Após a confirmação diagnóstica, os pacientes foram submetidos a tratamento antitrombótico com anticoagulante ou anti-agregante plaquetário. A escolha entre os tratamentos foi feita pela equipe de Neurologia Vascular de cada hospital. Os pacientes com evento hemorrágico não receberam nenhuma medicação antitrombótica e alguns foram submetidos à terapia endovascular.

Prognóstico - Os pacientes foram avaliados clinicamente aos 6 meses, sendo submetidos à escala modificada de Rankin, Índice de Barthel e escala de AVC do NIH. Como prognóstico favorável foi definido valor na escala de AVC do $\mathrm{NIH} \leq 1$, escala modificada de Rankin $\leq 1$ e Índice de Barthel $\leq 95$ aos 6 meses de seguimento.

Todos os pacientes realizaram angioRM de controle aos 6 meses para documentação de recanalização arterial e pesquisa de recorrência. Recanalização arterial foi definida como a normalização da artéria ou irregularidade, com estenose arterial residual menor que $30 \%$ em pacientes que apresentavam estenoses importantes $(>70 \%)$ ou oclusão no exame inicial ${ }^{12}$. A recorrência foi caracterizada como sintomática ou assintomática (alteração na angioRM aos 6 meses de seguimento na ausência de sinais e sintomas).

\section{RESULTADOS}

Dos 66 pacientes participantes do estudo, 42 apresentaram DAV e 24 DAC. Dissecção de múltiplas artérias foi observada em 4 pacientes.

Dados demográficos - Do total de pacientes, 53\% eram do sexo masculino. Houve predomínio de homens entre pacientes com DAV $(64 \%)$, enquanto $33 \%$ dos pacientes com DAC era do sexo masculino $(p=0,02)$. A média de idade dos pacientes foi 41,7 anos 
(20 a 62 anos). Observou-se predomínio importante de pacientes brancos, representando $82 \%$ do total. A distribuição étnica encontrada foi de 54 brancos, 11 afro-brasileiros e apenas 1 asiático.

Fatores de risco cardiovasculares - A Tabela 1 mostra a prevalência de fatores de risco cardiovasculares na população estudada.

Fatores predisponentes - A Tabela 2 exibe os fatores predisponentes de DEACV identificados. A presença de doença auto-imune foi observada em 2 pacientes.

Quadro clínico - A maioria dos pacientes (82\%) teve acidente vascular cerebral isquêmico (AVCI), 9\% apresentaram hemorragia subaracnóidea (HSA) e 6 não apresentaram evento neurovascular focal associado.

A Tabela 3 compara a DAC à DAV em relação às variáveis analisadas no estudo.

Diagnóstico por neuroimagem - TC de crânio foi realizada em todos os pacientes à admissão, mostrando-se alterada em $24 \%$ dos pacientes com DAV e $50 \%$ com DAC. A TC identificou HSA em 3 pacientes, todos com DAV. Nos outros 3 pacientes com HSA o diagnóstico foi confirmado através de análise do líquido cefalorraquidiano.

RM de encéfalo foi realizada em 32 pacientes com DAV, apresentando alterações em 29 (91\%). A anormalidade mais freqüente na DAV foi isquemia em região dorso-lateral bulbar, presente em 22 pacientes $(76 \%)$, e na DAC foi isquemia cerebral no território distal da artéria cerebral média.

Todos os pacientes foram submetidos a USG com Doppler. Em $62 \%$ dos pacientes com DAC o exame mostrou-se alterado, sendo estenose hemodinamica-
Tabela 1. Fatores de risco cardiovasculares nos casos de DAC e DAV.

\begin{tabular}{lcclccc}
\hline Variável & \multicolumn{3}{c}{ DAC } & & \multicolumn{3}{c}{ DAV } \\
\cline { 2 - 3 } \cline { 6 - 7 } & $\mathrm{N}$ & $\%$ & & $\mathrm{~N}$ & $\%$ \\
\hline Hipertensão arterial & 19 & 45 & & 6 & 25 \\
Tabagismo & 18 & 43 & & 16 & 67 \\
Etilismo & 14 & 33 & & 9 & 37 \\
Dislipidemia & 11 & 26 & & 8 & 33 \\
Diabetes & 1 & 2 & & 2 & 8 \\
Total & 42 & 64 & & 24 & 36 \\
\hline
\end{tabular}

Tabela 2. Fatores predisponentes nos casos de DAC e DAV.

\begin{tabular}{lcclccc}
\hline Variável & \multicolumn{3}{c}{ DAV } & & \multicolumn{2}{c}{ DAC } \\
\cline { 2 - 3 } \cline { 6 - 7 } & $\mathrm{N}$ & $\%$ & & $\mathrm{~N}$ & $\%$ \\
\hline Enxaqueca & 23 & 55 & & 11 & 46 \\
Evento menor precipitante & 14 & 33 & & 10 & 42 \\
Infecção recente & 10 & 24 & & 3 & 12 \\
Total & 42 & 64 & & 24 & 36 \\
\hline
\end{tabular}

mente significativa a alteração mais encontrada. Em apenas 2 casos de DAV ( $5 \%$ ) a USG estava alterada.

AngioRM foi realizada em $74 \%$ dos pacientes. Esse exame mostrou-se alterado em $94 \%$ dos pacientes com DAC e $81 \%$ com DAV. Angiografia cerebral com cateterismo foi realizada em 59 pacientes $(89 \%$ do total, sendo 21 com DAC e 38 com DAV). Quanto ao segmento arterial comprometido, $76 \%$ dos pacientes com DAC apresentaram envolvimento dos segmentos $\mathrm{C}_{1}$ e $\mathrm{C}_{2}$ (proximais) da artéria carótida interna e $79 \%$ dos pacientes com DAV apresentaram alteração no segmentos $V_{3}$ e $V_{4}$ da artéria vertebral. Dentre os pacientes com DAV, a presença de dilatação aneurismática foi observada em 6 pacientes (16\%). A angio-

Tabela 3. Comparação entre aspectos da DAC e DAV.

\begin{tabular}{|c|c|c|}
\hline Variável & DAC & DAV \\
\hline $\begin{array}{l}\text { Tempo médio entre início dos sintomas e } \\
\text { chegada ao hospital }\end{array}$ & $\begin{array}{c}6 \text { horas } \\
\text { (30 minutos a } 48 \text { horas) }\end{array}$ & $\begin{array}{c}9 \text { horas } \\
\text { (40 minutos a } 8 \text { dias) }\end{array}$ \\
\hline Valor médio na escala do NIH à admissão & $\begin{array}{c}8 \\
(0-21)\end{array}$ & $\begin{array}{c}3 \\
(0-9)\end{array}$ \\
\hline Quadro clínico mais freqüente & $\begin{array}{l}\text { Cefaléia, seguida por } \\
\text { déficit motor }(83 \%)\end{array}$ & $\begin{array}{c}\text { Cefaléia, seguida de } \\
\text { síndrome de Wallemberg }\end{array}$ \\
\hline Outros achados & $\begin{array}{l}\text { Síndrome de Horner }(37 \%) \text {, } \\
\text { paralisia de nervo craniano } \\
(17 \%) \text {, infarto retiniano }(8 \%)\end{array}$ & Vertigem $(60 \%)$ \\
\hline $\begin{array}{l}\text { Intervalo entre cefaléia e sinal ou } \\
\text { sintoma subseqüente }\end{array}$ & $\begin{array}{c}\text { 3,4 dias } \\
\text { (1 hora a } 1 \text { semana) }\end{array}$ & $\begin{array}{c}2,7 \text { dias } \\
\text { (1 hora a } 1 \text { semana) }\end{array}$ \\
\hline AVC hemorrágico & Nenhum paciente & HSA em 6 pacientes ( $14 \%)$ \\
\hline
\end{tabular}




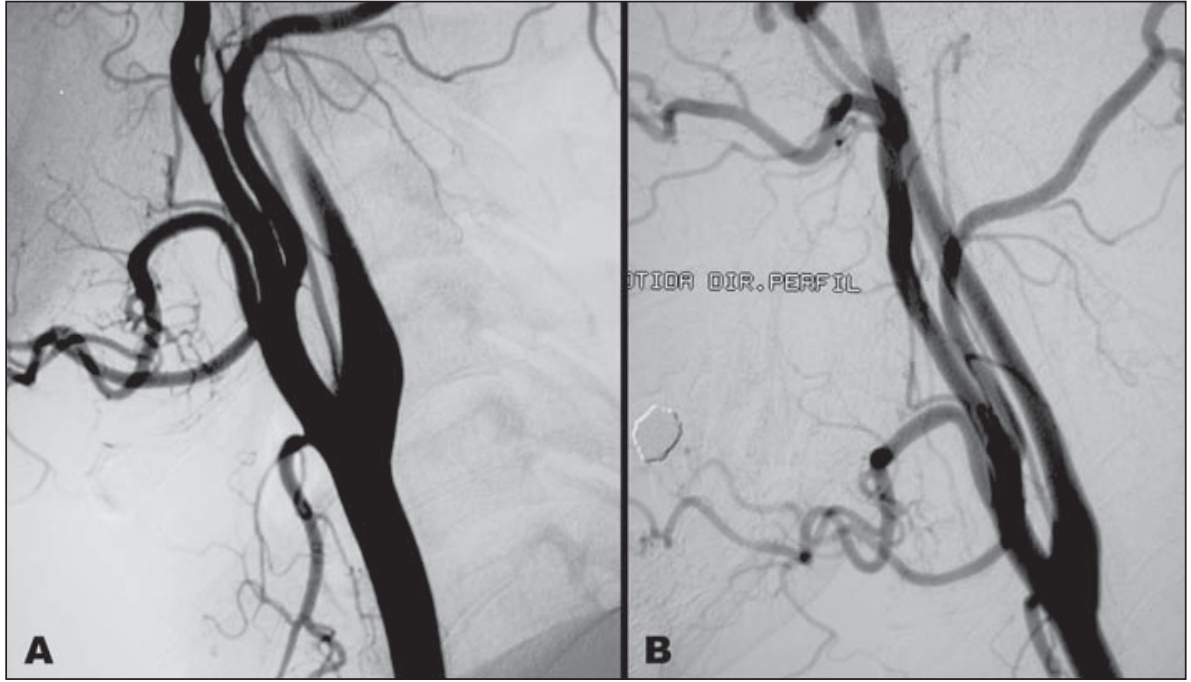

Figura. Paciente do ambulatório de Neurologia Vascular da EPM-Unifesp com dissecção espontânea de artéria carótida interna direita (A) que apresentou recanalização espontânea em 6 meses (B). grafia cerebral com cateterismo demonstrou fibrodisplasia em 14 pacientes (24\%). A RM com cortes axiais em T1 com supressão de gordura foi realizada em 19 pacientes (6 com DAC e 13 com DAV). Em 5 (83\%) pacientes com DAC e 6 ( $46 \%)$ com DAV o exame evidenciou o "sinal da dupla luz".

Tratamento - Em relação ao tratamento administrado, 35 pacientes (58\%) receberam anticoagulação nos primeiros 6 meses após o diagnóstico. Os demais pacientes com $\mathrm{AVCl}$ receberam $300 \mathrm{mg} /$ dia de AAS. Os 6 pacientes com HSA não receberam tratamento antitrombótico. Tratamento invasivo com procedimento endovascular foi realizado em 3 pacientes com DAV que apresentaram HSA e dilatação aneurismática associada. Nenhum paciente recebeu tratamento trombolítico.

Prognóstico - Em 6 meses de seguimento, 49 pacientes (74\%) apresentaram prognóstico favorável. Recanalização arterial foi detectada em 29 (53\%), sendo mais freqüente nos pacientes anticoagulados. A Figura mostra o exame de imagem de um dos pacientes nos quais ocorreu recanalização. Recidiva clínica ou no exame de imagem de controle ocorreu em 3 pacientes. Todos apresentavam DAV e a recidiva ocorreu na artéria vertebral contralateral. Durante o seguimento, um paciente foi a óbito, sendo a causa da morte não estabelecida.

\section{DISCUSSÃO}

Os hospitais selecionados são serviços terciários de ensino e de livre acesso, que atendem pacientes com AVC de todos os grupos étnicos e classes sociais. Contudo, observou-se nítido predomínio de pacientes brancos com DEACV, com valor bem acima do percentual de brancos na população brasileira e dos nossos hospitais ${ }^{13}$. Dados do censo brasileiro de 2001, mostram que $54 \%$ da população brasileira é composta por brancos ${ }^{14}$.

A média de idade dos pacientes foi 41,7 anos (20 a 62 anos), compatível com dados da literatura que consideram a DEACV como uma doença de jovens com pico na quinta década de vida ${ }^{15-17}$.

Não houve diferença entre o sexo dos pacientes envolvidos. Esse dado foi compatível com estudos prévios $^{15}$, porém observou-se maior freqüência de pacientes do sexo masculino com DAV, que pode estar associada a aspectos culturais e profissionais, com maior exposição de determinados pacientes a eventos precipitantes em atividades esportivas ou relacionadas ao trabalho.

Os estudos anteriores ainda permanecem inconclusivos quanto ao papel dos fatores de risco cardiovasculares na DEACV ${ }^{18}$. Nossos resultados são condizentes com essa informação, exceto pelo fato de $51 \%$ dos pacientes serem tabagistas. $O$ tabagismo pode predispor à fragilidade vascular favorecendo a ocorrência de DEACV, como sugerido nos casos de HSA decorrente de aneurismas intracranianos ${ }^{19}$. Alguns estudos demonstraram taxas mais elevadas dos fatores de risco cardiovasculares e este fato pode estar relacionado à inclusão de pacientes com dissecção de placas ateroscleróticas nesses estudos ${ }^{20}$.

A presença de história prévia de enxaqueca nos pacientes com DEACV é descrita desde os primeiros estudos dessa doença ${ }^{21}$. Estudos prévios demonstraram que pacientes com DEACV apresentam mais freqüentemente história prévia de enxaqueca quando 
comparados a pacientes com AVC de outra etiologia22. Nosso estudo reforçou esta informação, pois mais da metade dos pacientes com DEACV apresentavam história prévia de enxaqueca à admissão.

Foi demonstrado que 24 pacientes (36\%) tinham um evento menor precipitante relacionado à DEACV, podendo haver relação entre o contato das artérias com as estruturas ósseas adjacentes levando a lesões leves e contínuas durante movimentos cervicais usuais ou pouco traumáticos.

Infecção respiratória recente foi referida por 13 dos nossos pacientes (20\%). Estudos anteriores demonstraram que até $32 \%$ dos pacientes com DEACV tiveram uma infecção recente ${ }^{23}$. A relação entre doenças auto-imunes e DEACV vem sendo recentemente discutida na literatura, particularmente a associação entre DEACV e doença tireoidiana ${ }^{24}$. Em nosso estudo, 2 pacientes tinham tireoidite de Hashimoto.

A DEACV é uma causa de AVC isquêmico e hemorrágico, porém ela pode apresentar-se sem que um desses eventos ocorra. Nosso estudo mostrou que a maioria, dos pacientes apresentou AVCI. Evento hemorrágico ocorreu somente em pacientes com DAV, sob a forma de HSA. Nos pacientes que não tiveram evento isquêmico ou hemorrágico, a presença de cefaléia e de sinais focais como síndrome de Horner foram essenciais para o diagnóstico. A possibilidade de DEACV sem evento associado aumenta as chances do diagnóstico não ser realizado, colaborando para o subdiagnóstico dessa entidade.

Cefaléia foi o sintoma mais comum nos pacientes com DEACV estudados. Esse achado confirma a alta freqüência desse sintoma, que em outros estudos variou de $55-95 \%{ }^{25}$. A cefaléia foi seguida, na maioria dos casos, por um déficit motor. O período de tempo entre a cefaléia e o sinal ou sintoma subseqüente pode constituir uma importante janela terapêutica nos pacientes com DEACV. A introdução da terapêutica antitrombótica nessa fase poderia evitar a ocorrência do evento isquêmico. A maior dificuldade consiste em selecionar os pacientes, através das características da cefaléia, que devem ser investigados para o diagnóstico de DEACV. A tríade constituída por síndrome de Horner, infarto retiniano e isquemia de circulação anterior descrita nos pacientes com DAC foi observada em apenas 2 casos (8\%). Consideramos que a ocorrência desses sintomas de forma isolada, associada a outros dados como a presença de cefaléia em pacientes brancos jovens com ausência de fatores de risco cardiovasculares e presença de fatores predisponentes, seja bem mais importante do que a ocorrência da tríade. A síndrome de Horner foi observada em 37\% de nossos pacientes com DAC, em concordância com estudos prévios já publicados ${ }^{18,21,24,26}$. A paralisia de nervos cranianos é também considerada um importante sinal focal nos casos de DAC ${ }^{27}$. Em nosso estudo $12 \%$ dos pacientes com DAC apresentaram paralisia de nervo craniano. Esse achado pode levar à suspeita de isquemia no território vértebro-basilar levando a confusão diagnóstica ${ }^{27}$.

Uma grande parte dos pacientes com DEACV estudados apresentaram lesão cerebral em áreas de fronteira arterial, o que colabora com o conceito de que os dois mecanismos de lesão mais comumente envolvidos nos pacientes com DEACV são o hemodinâmico e o embólico arterio-arterial ${ }^{28}$. Dentre os pacientes com DAV, houve predomínio de lesão na porção dorso-lateral bulbar. Esta topografia foi também observada em outros estudos ${ }^{29}$.

A RM com cortes finos axiais em T1 com supressão de gordura mostrou-se alterada em mais da metade dos pacientes avaliados. Essa técnica possibilita o diagnóstico por demonstrar o "sinal da dupla luz", patognomônico da doença, porém sua sensibilidade depende do momento da realização do exame ${ }^{12}$. Considera-se que o momento ideal para o diagnóstico através desta técnica seja entre a primeira e a segunda semana após a DEACV. A RM possibilitou o diagnóstico na grande maioria dos pacientes submetidos a esse exame, reforçando a importância dos métodos de imagem não invasivos na avaliação dos pacientes com DEACV. A USG mostrou-se alterada em uma porcentagem muito baixa dos pacientes com DAV submetidos a esse exame. Com base nesse dado não devemos limitar a esse método a investigação dos casos suspeitos de DAV.

A angiografia cerebral com cateterismo é o método considerado padrão-ouro para o diagnóstico de DEACV. Em nossos pacientes, esse exame teve importância maior nos casos de DAV com dilatação aneurismática e nos pacientes em que a angioRM foi inconclusiva. A angiografia com cateterismo pode favorecer na determinação da etiologia da dissecção, como nos casos em que houve associação com fibrodisplasia.

Não há dados na literatura que suportem o uso de antitrombóticos, ou que demonstrem diferenças na evolução dos pacientes antiagregados ou anticoagulados $^{30}$. Desde uma publicação da década de 70 há tendência em utilizar anticoagulante oral nesses pacientes ${ }^{21}$. Em nosso estudo, $58 \%$ dos pacientes que receberam medicação antitrombótica foram antico- 
agulados nos primeiros 6 meses. Apesar de não ter havido randomização prévia, os pacientes anticoagulados apresentaram maior taxa de recanalização. Esse dado merece avaliação em novos estudos. Apesar de muitos pacientes na admissão, apresentarem tempo de início dos sinais e sintomas inferior a 3 horas, nenhum paciente recebeu tratamento trombolítico. A pontuação baixa na escala de AVC do NIH e a suspeita de HSA decorrente de cefaléia súbita, foram os principais critérios de exclusão para esse tratamento.

A DEACV é considerada por alguns autores como uma doença de bom prognóstico com taxa de recidiva baixa ${ }^{3}$. Neste estudo $74 \%$ dos pacientes tiveram prognóstico favorável em 6 meses. Apenas 1 paciente faleceu. A taxa de recanalização foi $53 \%$, ficando abaixo do observado em alguns estudos que demonstraram taxas de até $90 \%{ }^{2}$. Recorrência sintomática ocorreu em 2 pacientes e recorrência assintomática em 1, sempre em outra artéria, resultado semelhante ao previamente relatado na literatura ${ }^{15}$. Casos mais graves de DEACV podem ir a óbito nos serviços de emergência antes mesmo do diagnóstico ser estabelecido, não sendo considerados nas avaliações de prognóstico dessa doença.

Em conclusão, as informações obtidas em nosso estudo com uma população brasileira com DEACV foram semelhantes às observadas em outras populações analisadas previamente. Observou-se um nítido predomínio de pacientes brancos não previamente demonstrado na literatura. O sexo masculino predominou entre os pacientes com DAV, podendo refletir a importância dos fatores sócio-econômicos e culturais na ocorrência dos eventos precipitantes nos diferentes grupos de pacientes com DEACV. A presença de cefaléia inicial seguida por um déficit neurológico focal em um paciente jovem, branco, tabagista, com história prévia de enxaqueca e com ausência de fatores de risco cardiovasculares deve levantar a suspeita diagnóstica de DEACV. O envolvimento de territórios arteriais distais nos exames de imagem, associado à pontuação baixa na escala de AVC do NIH à admissão e ao prognóstico favorável apresentado pelos pacientes, levanta dúvidas quanto à relação risco-benefício dos tratamentos mais agressivos nos pacientes com DEACV. Esse estudo sugeriu que a RM com angioRM parece ser o exame inicial mais adequado na investigação dos pacientes com DEACV.

\section{REFERÊNCIAS}

1. De Bray JM, Lhoste P, Dubas F, Emile J, Saumet JL. Utrasonic features of extracranial carotid dissection: 47 cases studied by angiography. J Ultrasound Med 1994;13:659-664.
2. Ducrocq X, Lacour JC, Debouverie M, Bracard S, Girard F, Weber M. Accidents vasculaires cérébraux ischémiques du sujet jeune: étude prospective de 296 patients âgés de 16 à 45 ans. Rev Neurol (Paris) 1999;155:575-582.

3. Schievink WI. Spontaneous dissection of the carotid and vertebral arteries N Engl J Med 2001;344:898-906.

4. Baungartner RW, Bogoussslavsky J, Caso V, Paciaroni M. Handbook on cerebral artery dissection. Vol 20. Basel: Karger, 2005.

5. Guillon B, Biousse V, Massiou H, Bousser M-G. Orbital pain as an isolated sign of internal carotid artery dissection: a diagnostic pitfall. Cephalalgia 1998;18:222-224.

6. III Diretrizes Brasileiras sobre Dislipidemias e de Prevenção da Aterosclerose do Departamento de Aterosclerose da Sociedade Brasileira de Cardiologia. Arq Bras Cardiol 2001;77(Suppl 3):S1-S48.

7. Reynolds K, Lewis B, Nolen JD, Kinney GL, Sathya B, He J. Alcohol consumption and risk of stroke: a meta-analysis. JAMA 2003;289:579-588.

8. Headache Classification Committtee of the International Headache Society. Classification and diagnostic criteria for headache disorders, cranial neuralgias and facial pain. Cephalalgia 1988;(Suppl 7):S1-S96.

9. Hart RG, Easton JD. Dissections of cervical and cerebral arteries. Neurol Clin 1983;1:155-182.

10. Grau AJ, Brandt T, Buggle F, et al. Association of cervical artery dissection with recent infection. Arch Neurol 1999;56:851-856.

11. Adams HP Jr, Davis PH, Leira EC, et al. Baseline NIH Stroke Scale score strongly predicts outcome after stroke: a report of the trial of org 10172 in acute stroke treatment (TOAST). Neurology. 1999;53:126-131.

12. Djouhri $\mathrm{H}$, Guillon B, Brunereau L, et al. MR angiography for the longterm follow-up of dissecting aneurysms of the extracranial internal carotid artery. Am J Roentgenol 2000;174:1137-1140.

13. Pieri A, Alves MM, Valiente RA, Massaro AR. Spontaneous cervical artery dissection in a multiethnic population. Cerebrovasc Dis 2006;21(Suppl 4):S1-S151.

14. Instituto Brasileiro de Geografia e Estatistica. In: http://www.ibge.gov.br

15. Schievink WI, Mokri B, O’Fallon WM. Recurrent spontaneous cervical artery dissection. N Engl J Med 1994;330:393-397.

16. Leys D, Moulin TH, Stojkovic T, Begey S, Chavot D, DONALD Investigators. Follow-up of patients with history of cervical artery dissection. Cerebrovasc Dis 1995;5:43-49.

17. Bassetti C, Carruzzo A, Sturzenegger M, Tuncdogan E. Recurrence of cervical artery dissection: a prospective study of 81 patients. Stroke 1996;27:1804-1807.

18. Mokri B, Sundt TM Jr, Houser OW, Piepgras DG. Spontaneous dissection of the cervical internal carotid artery. Ann Neurol 1986;19:126-138.

19. Connolly ES Jr, Choudhri TF, Mack WJ, et al. Influence of smoking, hypertension, and sex on the phenotypic expression of familial intracranial aneurysms in siblings. Neurosurgery 2001;48:64-69.

20. Campos CR, Evaristo EF, Yamamoto FI, Puglia P, Lucato LT, Scaff M Spontaneous cervical carotid and vertebral arteries dissection: study of 48 patients. Arq Neuropsiquiatr 2004;62:492-498.

21. Fisher CM, Ojemann RG, Roberson GH. Spontaneous dissection of cervico-cerebral arteries. Can J Neurol Sci 1978;5:9-19.

22. Pezzini A, Granella F, Grassi M, et al. History of migraine and the risk of spontaneous cervical artery dissection. Cephalalgia 2005;25:575-580.

23. Guillon B, Berthet K, Benslamia L, Bertrand M, Bousser MG, Tzourio C. Infection and the risk of spontaneous cervical artery dissection: a case control study. Stroke 2003;34:79-81.

24. Pezzini A, Delztto E, Matizzioti G, et al. Thyroid autoimmunity and spontaneous cervical artery dissection. Stroke 2006;37:2375-2377.

25. Biousse V, D'Anglejan-Chatillon J, Massiou H, Bousser M-G. Head pain in non-traumatic carotid artery dissection: a series of 65 patients. Cephalalgia 1994;14:33-36.

26. Silbert PL, Mokri B, Schievink WI. Headache and neck pain in spontaneous internal carotid and vertebral artery dissections. Neurology 1995; 45:1517-1522.

27. Bravo-Rodriguez FA, Delgado Acosta F, Cano Sanchez A, Ramos Gomez MJ, Ochoa-Sepulveda JJ, Vida Lopez JM. Paralisis de pares craniales bajos como forma de presentacion de la diseccion de la artéria carótida interna. Rev Neurol 2002;34:754-757.

28. Koch S, Rabistein AA, Romano JG, Forteza AA. Diffusion-weighted magnetic resonance imaging in internal carotid artery dissection. Arch Neurol 2004;61:510-512.

29. Vilela P, Goulao A. Dissecções arteriais cervicais e intracranianas: revisão da apresentação aguda clinica e imaginologica de 48 casos. Acta Med Port 2003;16:155-164.

30. Lyrer P, Engelter S. Antithrombotic drugs for carotid artery dissection. Stroke 2004;35:613-614 